\title{
Catalytic Degradation of 4-Nitrophenol in Polluted Water by Three-Dimensional Gold Nanoparticles/Reduced Graphene Oxide Microspheres
}

\author{
Ning Li, ${ }^{1,2}$ Fuhua Zhang, ${ }^{1,2}$ Hua Wang ${ }^{2^{*}}$ and Shifeng $\mathrm{Hou}^{1,2^{*}}$
}

Microspheres as catalysts catalyst supports have attracted a great deal of attention over the last two decades. Here, gold nanoparticles (Au-NPs) encapsulated within the reduced graphene oxide microspheres (rGO microspheres) were prepared by using a spray-drying method. The Au-NPs were distributed evenly through the rGO microsphere composite. The mass content of Au-NPs in the rGO microspheres composite reached $30.3 \%$, and the average size of the Au-NPs was $10.5 \pm 2.5 \mathrm{~nm}$. The morphologies and structural characteristics of the materials were determined by scanning electron microscopy (SEM), transmission electron microscopy (TEM), high-resolution transmission electron microscopy (HRTEM) and X-ray diffraction (XRD) patterns. The as-prepared composite was used to catalyze the reduction of 4-nitrophenol to 4-aminophenol in the presence of excess sodium borohydride and exhibited high catalytic activity. The catalysis process was monitored by UV spectroscopy, and the first order rate constant of the catalytic reaction $\mathrm{K}$ was $1.96 \times 10^{-3} \mathrm{~s}^{-1}$, and the activation energy $\mathrm{E}_{\mathrm{a}}$ was found to be $3.5 \times 10^{4} \mathrm{~J} / \mathrm{mol}$. The catalytic experiment results indicated the $\mathrm{Au} / \mathrm{rGO}$ microspheres prepared have a great application for friendly environment aspects.

Keywords: Gold Nanoparticles; Reduced Graphene Oxide Microspheres; 4-nitrophenol; Catalytic Reduction

Received 8 January 2019, Accepted 15 March 2019

DOI: $10.30919 / \mathrm{es} 8 \mathrm{~d} 509$

\section{Introduction}

In recent years, the treatment technique of the pollution of water by phenol compounds has attracted widespread attention. Industrial production of pesticides, paints, and drugs produces 4-nitrophenol (4$\mathrm{NP}$ ), which is both difficult to degrade and toxic. 4-aminophenol (4AP) is a catalytic product of 4-NP and is less toxic than 4-NP, and thus to convert 4-NP into 4-AP is an ideal choice. ${ }^{1-3}$ At present, the commonly used methods for removing 4-NP are adsorption, microbial degradation, photocatalytic degradation, electrocoagulation and electrochemical methods. ${ }^{47}$ Although the above methods have certain effects on the removal of 4-NP, they have some limitations, such as inefficient degradation, slow degradation and secondary pollution. Additionally, the operating costs, reaction conditions and equipment also have higher requirements. The hydrogenation method has the advantages of being mild, efficient, rapid, and environmentally friendly, meeting the "green chemistry" concept. ${ }^{8-10}$ Commonly used catalysts include noble metals ( $\mathrm{Au}, \mathrm{Pt}, \mathrm{Pd}, \mathrm{Ru}, \mathrm{Rh}$ ) and non-precious metals (Ag, $\mathrm{Cu}, \mathrm{Ni}, \mathrm{Fe}, \mathrm{Mn}$ ). ${ }^{11-20} \mathrm{Au}$ nanoparticles (Au-NPs) have more efficient catalytic activity under mild conditions. ${ }^{21}$ The conversion of 4-NP to 4AP catalyzed by Au-NPs in sodium borohydride solution $\left(\mathrm{NaBH}_{4}\right)$ has been extensively studied. However, Au-NPs have a large specific surface area and very high surface energy, which lead to agglomeration. Once Au-NPs agglomerate, their catalytic activity decreases. While

\footnotetext{
'School of Chemistry and Chemical Engineering, Shandong University, Jinan, Shandong 250100, China

2 National Engineering and Technology Research Center for Colloidal Materials, Shandong University, Jinan, Shandong 250100, China

*E-mail: h.wang@sdu.edu.cn
}

ensuring high catalytic activity sites, it is extremely important to prevent the agglomeration of Au-NPs. ${ }^{22-25}$ To inhibit the agglomeration of Au$\mathrm{NPs}$, they were loaded on carriers of various morphologies and compositions. Spherical nanocomposites prepared by the spherical carrier have the advantages of a high specific surface area, excellent diffusion properties and easily modified surfaces on the spherical nanostructures. ${ }^{26-27}$ Catalytic materials prepared with spherical carriers not only exhibit high activity and high stability to target reactions but also are recyclable, while the inorganic type of spherical carrier also has high temperature resistance, anti-aging properties, etc. ${ }^{28-29}$ There are a number of spherical substrates supported Au-NPs that catalyze the reduction of 4-NP. For example, Dong et $a l^{30}$ synthesized Au-NPs loaded with polysilsesquioxane, which was used as catalyst in the reduction of 4-NP. There was no by-product in the reduction of 4-NP, and the conversion rate reached $97.8 \%$ in 60 minutes. After centrifugal recovery and 10 cycles, the conversion rate remained at $95.1 \%$.

Graphene is a newly discovered two-dimensional carbon material with a very large surface area, good electrical conductivity, and high electron transport capacity. ${ }^{31-32}$ The Au-NPs dispersed in graphene can not only guarantee that $\mathrm{Au}-\mathrm{NPs}$ reassemble together but also use a large specific surface of the graphene to bring the material into full contact with the waste liquid. For example, Vellaichamy et al. $^{33}$ synthesized AuNPs@RGO nanosheets. Although it has a good reducing effect on 4nitrophenol, but still cannot solve the problem of reduced catalytic degradation efficiency due to agglomeration of AuNPs. Further, the problem with graphene material is that the two-dimensional graphene sheet has a large surface area to easily reunite into a bulk piece by Van der Waals force and $\pi-\pi$ stacking. ${ }^{34}$ The two-dimensional graphene being made into three-dimensional can be a good solution to this problem.

In this study, we fabricate Au-NPs supported on reduced graphene (Au-NPs/rGO) microspheres to form composites for the efficient 
catalytic degradation of 4-NP. The Au-NPs/rGO microsphere composites were prepared by spray-coating followed by hightemperature thermal reduction. It was found that Au-NPs/rGO microsphere composites showed an outstanding catalytic performance towards 4-nitrophenol to 4-aminophenol. AuNPs/rGO microspheres are effective catalyst for 4-NP reduction in the presence of $\mathrm{NaBH}_{4}$, and the catalytic process was detected by UV-Visible spectrum. The catalytic effect and recyclability of Au-NPs/rGO microspheres for 4-NP in polluted water are superior to those of conventional catalyst materials.

Compared with Au-NPs/rGO lamellae material, several advantages of as-prepared $\mathrm{Au}-\mathrm{NPs} / \mathrm{rGO}$ microspheres to catalyze the reduction of 4-Nitrophenol are as follows. (i) The high specific surface area of the three-dimensional microsphere structure with the cell as a natural model provides more active sites for the Au-NPs, which facilitates sufficient contact between the catalyst and the waste liquid and the progress of the catalytic process. (ii) The microsphere structure can effectively stabilize and disperse the gold nanoparticles, stabilize the particles against aggregation, and increase the catalytic reduction rate. (iii) Compared with ordinary bulk two-dimensional graphene, the threedimensional graphene microspheres have small volume, excellent dispersion performance and stability, and are beneficial to improve the stability and recycling of the graphene-based catalyst. ${ }^{35}$

\section{Experimental section}

\subsection{Chemicals}

Graphite oxide (GO) was provided by Jining Leadernano Co., Ltd. (Jining, China). 4-Nitrophenol (4-NP) and sodium borohydride were supplied by Sinopharm Chemical Reagent Co., Ltd. (Shanghai, China). Chloroauric acid $\left(\mathrm{HAuCl}_{4} \cdot 4 \mathrm{H}_{2} \mathrm{O}\right)$ and trisodium citrate were provided by the Sinopharm Group Chemical Reagent Co., Ltd. All reagents were analytical grade and were used directly without further treatment. All aqueous solutions were prepared with deionized water with a resistivity of $18.25 \mathrm{M} \Omega \cdot \mathrm{cm}$.

\subsection{Sample preparation}

\subsubsection{Preparation of gold nanoparticles (Au-NPs)}

The chloroauric acid was used to prepare into a $24.28 \mathrm{mM} \mathrm{HAuCl}_{4}$ solution. In a typical synthesis, $100 \mathrm{~mL}$ of the $\mathrm{HAuCl}_{4}$ solution was placed in a three-necked flask and heated to reflux for 3 minutes. Then $80 \mathrm{~mL}$ of a $10 \%$ trisodium citrate solution containing $0.34 \mathrm{~g}$ of polyvinylpyrrolidone (PVP) was quickly injected and the solution was further boiled for 10 minutes. Magnetic stirring was maintained for the entire reaction period, until cool to room temperature, at which point the solution was stored in the refrigerator at $4{ }^{\circ} \mathrm{C}$ for use.

\subsubsection{Synthesis of $A u-N P s / r G O$ microspheres and $A u-N P s / r G O$ lamellae composites}

The Au-NPs/rGO microspheres were synthesized by using a spraydrying method as illustrated in previously reported procedures with slight modification. ${ }^{36}$ In a standard procedure, $5.0 \mathrm{mg} / \mathrm{mL}$ GO solution was mixed with the Au-NPs solution and sonicated for 3 hours to ensure even mixing. The $\mathrm{Au}-\mathrm{NPs} / \mathrm{GO}$ precursor solution, with a mass ratio of $\mathrm{HAuCl}_{4}$ to $\mathrm{GO}$ of 1:1, was spray-dried and collected to heat at $500{ }^{\circ} \mathrm{C}$ in a tube furnace under nitrogen atmosphere. Over the thermal reduction process, the samples were cooled down to room temperature naturally. Products were rinsed with ultra-pure water several times, then dried and stored for future usage. The Au-NPs/rGO lamellae material was synthesized in the manner described above, except that the spraydrying method was changed into the freeze-drying method.

\subsection{Apparatus}

The BILON-6000Y spray-dryer (Shanghai Bilang Instrument Manufacturing Co. Ltd., China) was used for spray-drying to obtain Au$\mathrm{NPs} / \mathrm{rGO}$ microspheres composites. The CF-16RN centrifuge (Hitachi kokl Co. Ltd, Tokyo Japan) was used for centrifugation to obtain a catalyzed solution. The morphology of the composites was obtained by a SU8010 microscope $(3.0 \mathrm{kV}$ scanning electron microscopy [SEM] HITACHI, Tokyo, Japan), a JEM-1011 microscope (200 kV transmission electron microscopy [TEM] JEOL, Tokyo, Japan) and a JEM-2100 microscope (200 kV high-resolution transmission electron microscopy [HRTEM] JEOL, Tokyo, Japan). The structural characteristics of the samples were characterized by filtered $\mathrm{Cu} \mathrm{K \alpha}$, $\lambda=0.154 \mathrm{~nm}$ X-ray diffraction (XRD) patterns and Raman spectrometer (PHS-3C, Horbin, France) with excitation of $633 \mathrm{~nm}$ laser. The UV spectrum was measured by a TU-1901 UV-Visible Spectrophotometer (PERSEE, Beijing, China). The content of the material components was obtained by measuring diamond TG / DTA thermogravimetric analysis (Perkin Elmer, America) using air as the process gas, a gas flow rate of $20 \mathrm{sccm}$, and a heating rate of $10{ }^{\circ} \mathrm{C} / \mathrm{min}$.

\subsection{Catalytic properties}

The catalytic reduction properties of the 4-nitrophenol with as-prepared catalyst were investigated by adding $0.1 \mathrm{~mL}$ of 4-nitrophenol aqueous solution $(4.0 \mathrm{mM})$ into $5.0 \mathrm{~mL}$ distilled water solution. A certain amount of AuNPs/rGO microsphere $(1.0 \mathrm{mg} / \mathrm{mL})$ was added to the solution, and then $0.4 \mathrm{~mL}$ of fresh $\mathrm{NaBH}_{4}$ aqueous solution $(0.12 \mathrm{M})$ was added to above solution and the stop-watch was used to immediately to record the reaction time. The solution was then filled quickly at a giving time or centrifuged for 2 minutes (14000 r/min), and the above solution was used to fill a cuvette for testing with UV-VIS.

During the catalytic reaction, 4-NP was converted to 4-AP, and then the absorbance of 4-nitrophenol at $400 \mathrm{~nm}$ will decrease. The absorbance of 4-nitrophenol at $400 \mathrm{~nm}$ is proportional to its concentration, so the concentration ratio of 4-nitrophenol at the giving time $\left(\mathrm{C}_{\mathrm{t}}\right)$ to the initial concentration $\left(\mathrm{C}_{0}\right), \mathrm{C}_{\mathrm{t}} / \mathrm{C}_{0}$ will be equal to the ratio of absorbance intensity at giving time $\mathrm{t}_{\mathrm{t}}\left(\mathrm{A}_{\mathrm{t}}\right)$ to that at start $\mathrm{t}_{0}\left(\mathrm{~A}_{0}\right)$. As a result, the progress of the reaction could be directly reflected by the change of the Ultraviolet absorption intensity of 4-NP and 4-AP.

\section{Results and discussions}

\subsection{Morphology and structure of the Au-NPs/rGO microspheres}

The detail morphology structures of the Au-NPs/rGO microspheres were depicted in Fig. 1. Fig. 1a, b and c depicted the SEM and TEM image of Au-NPs/rGO microspheres, it can be seen that a well-defined microsphere structure were obtained with rGO sheet as the microsphere and the Au-NPs distributed within the microsphere entirely. From Fig. $1 \mathrm{~b}$, the Au-NPs with a lattice spacing of $0.23 \mathrm{~nm}$ (inset of Fig. 1d) were found to be uniformly dispersed in the graphene material, and Fig. 1d showed the size of the Au-NPs, which average to $(10.5 \pm 2.5) \mathrm{nm}$ (inset of Fig. 1d). As a control experiment, the Au-NPs/rGO materials prepared by the freeze-drying method have sheet structures (shown in Figs S1a and b). To further demonstrate the uniform distribution of AuNPs in Au-NPs/rGO microspheres, we have performed mapping of the image in Fig. 2. It can be seen that the Au-NPs are distributed evenly throughout the microsphere without aggregation.

In order to further determine the successful synthesis of Au-NPs/rGO microspheres, Raman and XRD were also implemented. Fig. 3 showed Raman spectra of GO, rGO, Au-NPs/rGO microspheres and Au$\mathrm{NPs} / \mathrm{rGO}$ lamellae. Au-NPs/rGO microspheres and Au-NPs/rGO lamellae have similar Raman peak characteristics corresponding to the 


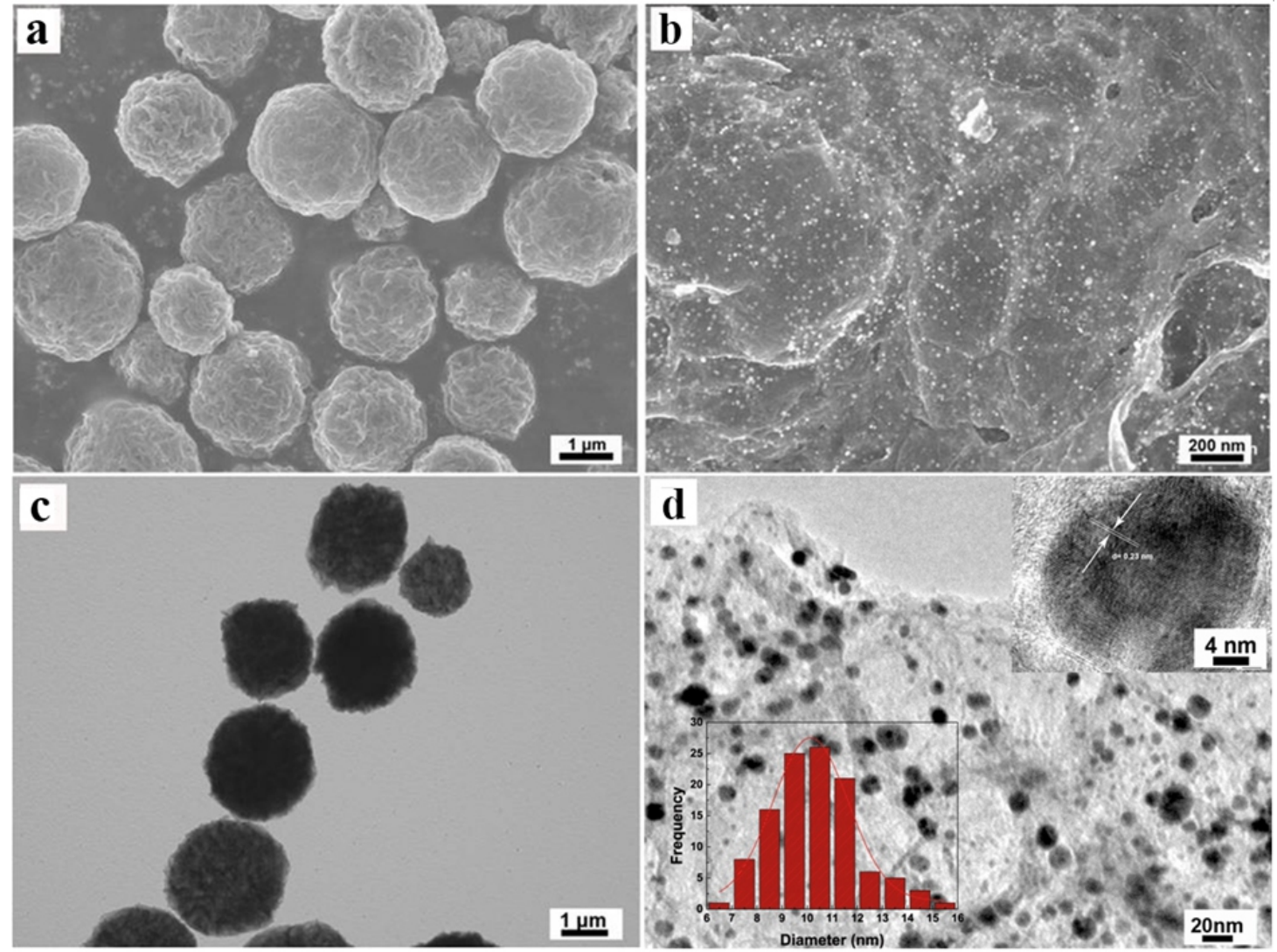

Fig. 1 (a), (b) SEM and (c) TEM images of Au-NPs/rGO microspheres, (d) HRTEM image of Au nanoparticles of Au-NPs/rGO microspheres, the inset is a single $\mathrm{Au}$ nanoparticle of $\mathrm{Au}-\mathrm{NPs} / \mathrm{rGO}$ microspheres and average particle size distribution.

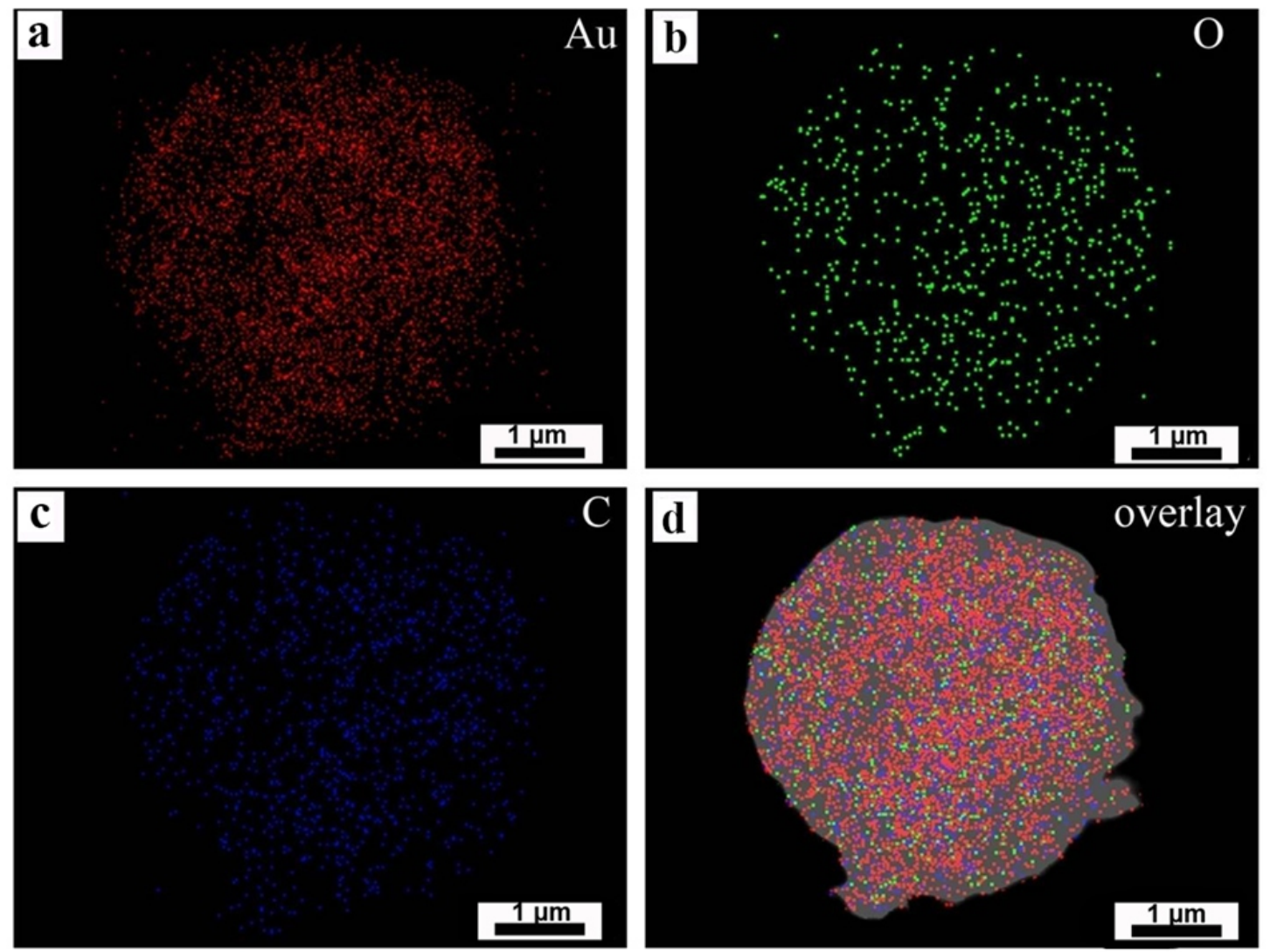

Fig. 2 The mapping of Au-NPs/rGO microspheres. 


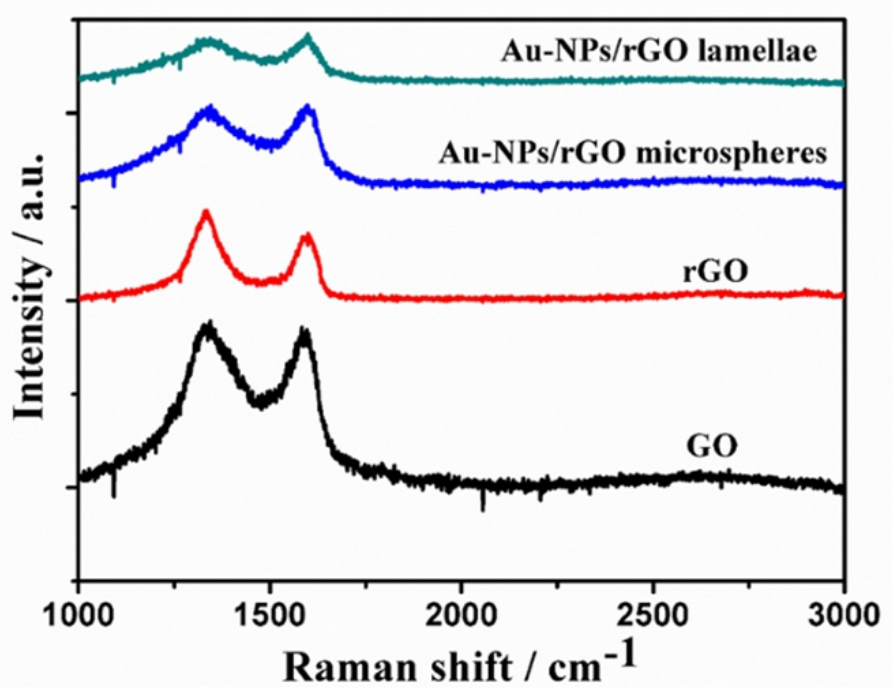

Fig. 3 Raman spectra of GO, rGO, Au-NPs/rGO microspheres, AuNPs/rGO lamellae.

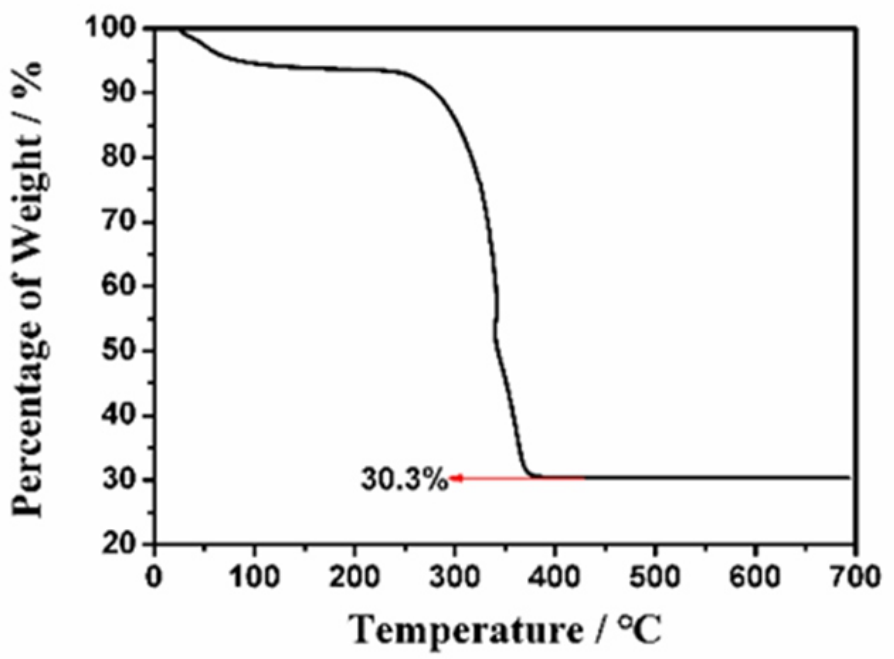

Fig. 5 Thermogravimetric curve of Au-NPs/rGO microspheres.

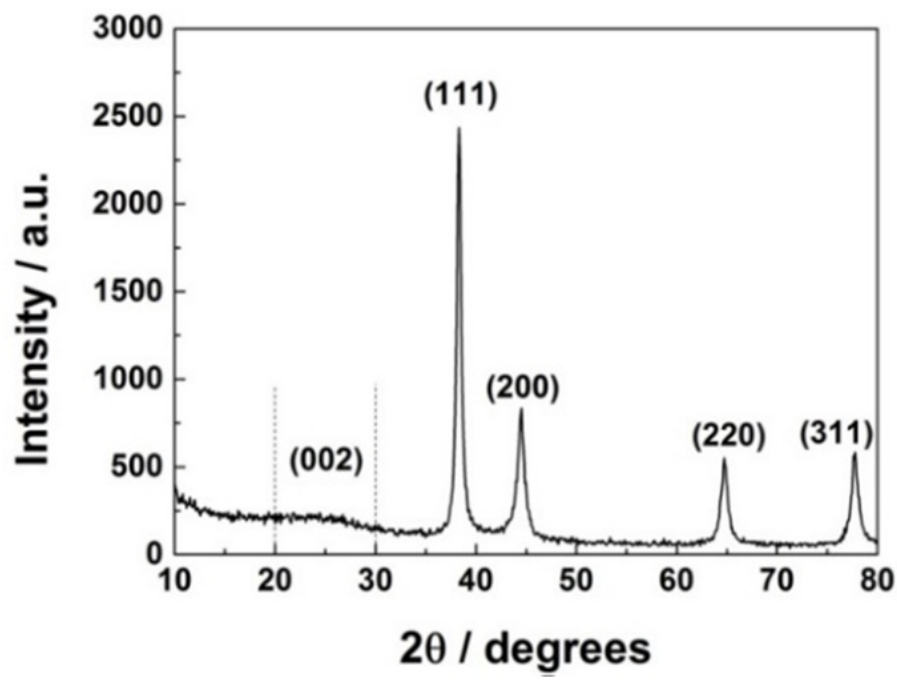

Fig. 4 The diffraction pattern of the Au-NPs/rGO microspheres.

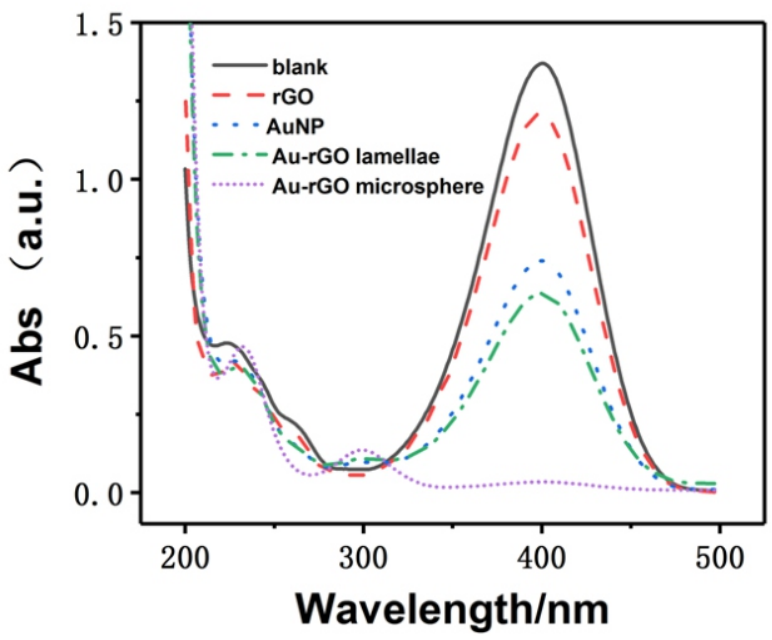

Fig. 6 UV spectrum of blank solution, rGO, Au-NPs, the Au-NPs/rGO lamellae, Au-NPs/rGO microspheres in $20.0 \mathrm{M} 4-\mathrm{NP}, 400.0 \mathrm{M} \mathrm{NaBH}_{4}$ at $298 \mathrm{~K}$ for 30 minutes.

Table 1 Specific surface area and pore structure of the Au-NPs/rGO materials studied.

\begin{tabular}{|c|c|c|c|c|c|}
\hline \multirow[b]{2}{*}{ Material } & \multirow{2}{*}{$\begin{array}{l}\text { BET surface } \\
\text { area }\left(\mathrm{m}^{2} \mathrm{~g}^{-1}\right)\end{array}$} & \multirow{2}{*}{$\begin{array}{c}\text { Pore } \\
\text { volume } \\
\left(\mathrm{cm}^{3} \mathrm{~g}^{-1}\right)\end{array}$} & \multicolumn{3}{|c|}{ t-Plot } \\
\hline & & & $\begin{array}{r}\text { Micropore } \\
\text { area }\left(\mathrm{m}^{2} \mathrm{~g}^{-1}\right)\end{array}$ & $\begin{array}{l}\text { External surface } \\
\text { area }\left(\mathrm{m}^{2} \mathrm{~g}^{-1}\right)\end{array}$ & $\begin{array}{l}\text { t-Plot micropore } \\
\text { volume }\left(\mathrm{cm}^{2} \mathrm{~g}^{-1}\right)\end{array}$ \\
\hline $\mathrm{Au}-\mathrm{NPs} / \mathrm{rGO}$ microspheres & 230.4 & 0.28 & 167.6 & 62.9 & 0.062 \\
\hline $\mathrm{Au}-\mathrm{NPs} / \mathrm{rGO}$ lamellae & 146.7 & 0.11 & 108.2 & 38.5 & 0.047 \\
\hline
\end{tabular}


D (1344 $\left.\mathrm{cm}^{-1}\right)$ and $\mathrm{G}\left(1583 \mathrm{~cm}^{-1}\right)$ bands of $\mathrm{GO}$ and $\mathrm{rGO}^{37}$ By

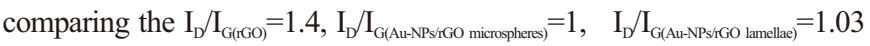
and peak shape curve, it can be roughly obtained that the addition of $\mathrm{Au}-\mathrm{NPs}$ has a certain influence on the Raman peak of Au-NPs/rGO microspheres and $\mathrm{Au}-\mathrm{NPs} / \mathrm{rGO}$ lamellae, indicating that the Au-NPs on the surface and internal nucleation of rGO will bring additional defects to the structure. Fig. 4 displayed the XRD pattern of Au-NPs/rGO microspheres, which showed four peaks at about 38.4, 44.6, 64.8, and $77.9^{\circ}$, corresponding to the (111), (200), (220), (311) crystal faces of the face-centred cubic structure of crystalline Au ((JCPDS file card no. 04-0784), respectively. And $20-30^{\circ}$ wide $\mathrm{C}$ peak indexed to the (002) crystal face of graphene. ${ }^{38}$ According to Scherrer's Formula, the average size of Au-NPs was about $11 \mathrm{~nm}$, which is consistent with the result of TEM.

The gold content of the synthesized material was further confirmed by the thermogravimetric analysis. A typical thermogravimetric curve in Fig. 5 showed that the content of gold nanoparticles in the composites was $30.3 \mathrm{wt} \%$, which was in accordance with the theoretical designed $33.3 \mathrm{wt} \%$, demonstrated that a high loading of non-agglomerated AuNPs within the graphene made by the spray-drying method.

The total pore volume and the Brunauer Emmett Teller (BET) specific surface area of Au-NPs/rGO microsphere are $0.28 \mathrm{~cm}^{3} \mathrm{~g}^{-1}$ and $230.4 \mathrm{~m}^{2} \mathrm{~g}^{-1}$, respectively, which are much higher than that of freezedried rGO sample (Table 1). Au-NPs/rGO microspheres have a large specific surface area, provide a larger contact site with 4-NP, and facilitate the uniform dispersion of Au-NPs to provide sufficient catalytic sites to increase the rate of catalytic degradation to 4-NP. The reason for the analysis may be that graphene microspheres are beneficial to maintaining a three-dimensional multi-space network, and the flaky graphene sample prepared by lyophilization tends to collapse the pores and fail to provide a high specific surface area.

\subsection{Catalytic reduction of 4-NP to 4-AP}

The catalytic properties of the catalyst toward the reduction of 4-NP in the excess of $\mathrm{NaBH}_{4}$ were then investigated by UV-vis spectra. The UV spectrum in Fig. 6 showed the catalytic process of rGO, Au-NPs, Au$\mathrm{NPs} / \mathrm{rGO}$ lamellae, and Au-NPs/rGO microspheres towards the reduction in $20.0 \mathrm{M}$ 4-NP, $400.0 \mathrm{M} \mathrm{NaBH}_{4}$ at $298 \mathrm{~K}$. The UV spectrum at $400 \mathrm{~nm}$ was the 4-NP ion absorption peak. With 4-NP conversion to 4-AP, the UV absorption peak at $400 \mathrm{~nm}$ gradually decreased, while the UV absorption peak associated with 4-AP appeared at $290 \mathrm{~nm}^{39}$ As we can see from the UV-vis spectrum, rGO did not have a catalytic effect as the blank solution, but there is a slight adsorption of 4-NP. The catalytic effect of Au-NPs/rGO lamellae was weaker than that of the $\mathrm{Au}-\mathrm{NPs}$ solution. However, the catalytic effect of Au-NPs /rGO microspheres was better than that of the Au-NPs solution.

To compare the catalytic effects of $\mathrm{Au}-\mathrm{NPs} / \mathrm{rGO}$ microspheres and $\mathrm{Au}-\mathrm{NPs} / \mathrm{rGO}$ lamellae, we placed $0.5 \mathrm{~mL}$ of Au-NPs/rGO microsphere suspension $(1.0 \mathrm{mg} / \mathrm{mL})$ into $0.1 \mathrm{~mL} 4.0 \mathrm{mM}$ 4-NP, $0.4 \mathrm{~mL} 120.0 \mathrm{mM}$ $\mathrm{NaBH}_{4}$, and $5 \mathrm{~mL}$ deionized water at $298 \mathrm{~K}$. The UV-vis spectra of the reaction process were depicted in Fig. $7 \mathrm{a}$ and $\mathrm{b}$. It can be seen from the Fig. 7 that the Au-NPs/rGO microsphere material can convert the 4-NP to 4-AP entirely within 28 minutes, and the Au-NPs/rGO lamellae material did so with at least 140 minutes. The catalyst received the electron donor $\mathrm{BH}_{4}^{-}$, then transferred to the electron acceptor of 4-NP, and thus the catalytic reaction occurred. ${ }^{40}$ Since $\mathrm{NaBH}_{4}$ was in excess, it could be considered that the reduction rate was independent of the $\mathrm{NaBH}_{4}$ content. $\mathrm{NaBH}_{4}$ was added to the 4-NP solution to form a 4-NP ion, and the color of the solution changed from pale yellow-green to dark yellow-green.

Fig. 7c was a linear correlation between $\ln \left(\mathrm{A}_{\mathrm{t}} / \mathrm{A}_{0}\right)$ and reaction time $(\mathrm{T})\left(\mathrm{A}_{t}\right.$ was the intensity of the absorption peak after a certain time $\mathrm{t}$, and $\mathrm{A}_{0}$ was the intensity of the absorption peak at the beginning), indicating that the reaction was a pseudofirst-order reaction. ${ }^{41}$ The Au$\mathrm{NPs} / \mathrm{rGO}$ microspheres had a pseudofirst-order rate constant of $\mathrm{K}_{\text {app }}=-$ $1.96 \times 10^{-3} \mathrm{~s}^{-1}$, which was much higher than the Au-NPs/rGO lamellae material's $\mathrm{K}_{\text {app }}=-0.36 \times 10^{-3} \mathrm{~s}^{-1 .}$ We also calculated the ratio of the rate constant $\mathrm{K}$ to the weight of Au-NPs in the catalyst, $\mathrm{K}_{\text {app }} / \mathrm{m}=\mathrm{K} / \mathrm{m}=12.94$ $\mathrm{s}^{-1} \mathrm{~g}^{-1}$. Table 2 showed a comparison of Kapp and Kapp/m of different catalytic systems for the reduction of 4-NP (298 K). It could be found that the Au-NPs/rGO microsphere prepared in this work had the highest catalytic activity. One reason was that the microsphere structures can improve the mass transfer as compared to the same mass of lamellae supported catalysts. And the microsphere structure of graphene provided specific surface area, which can be effective for adsorbing 4-NP by $\pi-\pi$ conjugation and the free space within the microsphere provided more space for the 4-NP to reach the catalytic sites. ${ }^{42-43}$ Another reason was that the microsphere structures can prevent the leaking and aggregation of gold nanoparticles, which can provide more catalytic activity sites to 4-NP and thus can stabilize the gold nanoparticles during the reaction process.

To test the influence of the amount of catalyst on the catalytic behavior, we added $0.25 \mathrm{~mL}, 0.5 \mathrm{~mL}$, and $0.75 \mathrm{~mL}$ of $\mathrm{Au}-\mathrm{NPs} / \mathrm{rGO}$ solution $(1.0 \mathrm{mg} / \mathrm{mL})$ to $6.0 \mathrm{~mL}$ reaction solution of $0.4 \mathrm{~mol} \mathrm{4-NP}$ and
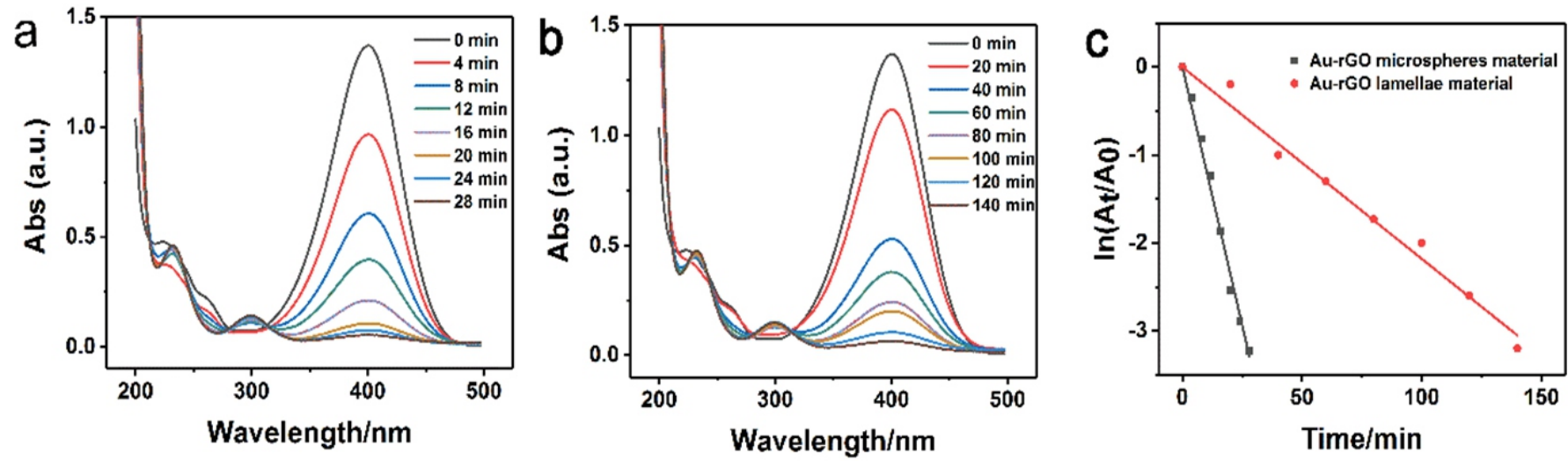

Fig. 7 UV spectrum of (a) Au-NPs/rGO microspheres; (b) Au-NPs/rGO lamellae in 0.4 mol 4-NP, 48.0 mol NaBH $\mathrm{H}_{4}$ at $298 \mathrm{~K}$; (c) the relation of $\mathrm{t}$ vis $\ln \left(\mathrm{A}_{1} / \mathrm{A}_{0}\right)$. 
$48.0 \mathrm{~mol} \mathrm{NaBH}_{4}$, and the results were depicted in Fig. 8. Fig. 8c shows a linear correlation between $\ln \left(\mathrm{A}_{\mathrm{l}} / \mathrm{A}_{0}\right)$ and $\mathrm{T}$ of $0.25 \mathrm{~mL}, 0.5 \mathrm{~mL}$, and $0.75 \mathrm{~mL}$ of microspheres $(1.0 \mathrm{mg} / \mathrm{mL})$. Pseudofirst-order rate constants were $\mathrm{K}_{0.25}=-1.25 \times 10^{-3} \mathrm{~s}^{-1}, \mathrm{~K}_{0.5}=-1.96 \times 10^{-3} \mathrm{~s}^{-1}$, and $\mathrm{K}_{0.75}=-3.13 \times 10^{-3} \mathrm{~s}^{-1}$. The corresponding $\mathrm{K}_{\text {app }} / \mathrm{m}$ is $\mathrm{K}_{0.25} / \mathrm{m}=16.5 \mathrm{~s}^{-1} \mathrm{~g}^{-1}, \mathrm{~K}_{0.5} / \mathrm{m}=12.94 \mathrm{~s}^{-1} \mathrm{~g}^{-1}$, $\mathrm{K}_{1.5} / \mathrm{m}=13.77 \mathrm{~s} \mathrm{~s}^{-1} \mathrm{~g}^{-1}$ respectively. It can be seen that $\mathrm{K}$ increased with the increasing concentration of catalyst. This was because as the amount of catalyst increases, the amount of catalysis site of the catalyst increased, leading to an increase in $\mathrm{K}$, but the reaction rate of per mass of AuNPs is basically unchanged, resulting in $\mathrm{K}_{\mathrm{app}} / \mathrm{m}$ staying constant roughly.

The effect of temperature on the reduction of 4-NP with $\mathrm{NaBH}_{4}$ in the presence of $\mathrm{Au}-\mathrm{NPs} / \mathrm{rGO}$ microspheres at different temperature was shown in Fig. 9a and b, and a linear correlation between $\ln \left(\mathrm{A}_{\mathrm{t}} / \mathrm{A}_{0}\right)$ and $\mathrm{T}$ across 273, 298 and $323 \mathrm{~K}$ displayed in Fig. 8c. The values of the pseudofirst-order rate constants were $\mathrm{K}_{273 \mathrm{~K}}=-0.34 \times 10^{-3} \mathrm{~s}^{-1}, \mathrm{~K}_{298 \mathrm{~K}}=$ $1.96 \times 10^{-3} \mathrm{~s}^{-1}$, and $\mathrm{K}_{323 \mathrm{~K}}=-3.55 \times 10^{-3} \mathrm{~s}^{-1}$. According to the relationship between temperature and pseudofirst-order rate constants, we can calculate the activation energy $\mathrm{Ea}=3.5 \times 10^{4} \mathrm{~J} / \mathrm{mol}$ by the Arrhenius equation.

The catalytic stability of the as-prepared Au-NPs/rGO microspheres was then examined. In a typical experiment, $0.5 \mathrm{mg}$ of the catalyst were added into $0.1 \mathrm{~mL} 4.0 \mathrm{mM}$ 4-NP solution at $298 \mathrm{~K}$. After 20 minutes,

Table 2 Comparison of $\mathrm{K}_{\text {app }}$ and $\mathrm{K}_{\text {app }} / \mathrm{m}$ of different catalytic systems for the reduction of 4-NP $(298 \mathrm{~K})$.

\begin{tabular}{|c|c|c|c|c|c|}
\hline Support & $\begin{array}{c}\text { Catalyst usage } \\
{[\mathrm{wt} \%]}\end{array}$ & $\begin{array}{l}\mathrm{NaBH}_{4} \\
\text { (equiv.) }\end{array}$ & $\mathrm{K}_{\mathrm{app}}\left(\mathrm{s}^{-1}\right)$ & $\begin{array}{l}\mathrm{K}_{\mathrm{app}} / \mathrm{m} \\
\left(\mathrm{s}^{-1} \mathrm{~g}^{-1}\right)\end{array}$ & Ref. \\
\hline $\mathrm{Au} / \mathrm{PTSC}$ & 33.50 & excess & $1.60 \times 10^{-4}$ & 3.34 & 44 \\
\hline graphene/PDA-Au Nps & - & - & $3.75 \times 10^{-3}$ & 3.0 & 45 \\
\hline $\mathrm{Fe}_{3} \mathrm{O}_{4} @ \mathrm{CeO}_{2} / \mathrm{Au}$ & 1.35 & 80 & $1.53 \times 10^{-3}$ & 56.67 & 46 \\
\hline hybrid $\mathrm{Au}-\mathrm{GO}$ nanocomposites & 62 & excess & $2.06 \times 10^{-3}$ & 8.37 & 47 \\
\hline $\mathrm{C} / \mathrm{Au} / \mathrm{TiO}_{2}$ & 0.86 & - & $8.7 \times 10^{-3}$ & 10.08 & 48 \\
\hline PS-AuNP & 4.7 & excess & $2.75 \times 10^{-4}$ & 9.7 & 49 \\
\hline $\mathrm{Au}-\mathrm{NPs} / \mathrm{rGO}$ microsphere & 30.3 & excess & $1.96 \times 10^{-3}$ & 12.94 & This work \\
\hline
\end{tabular}
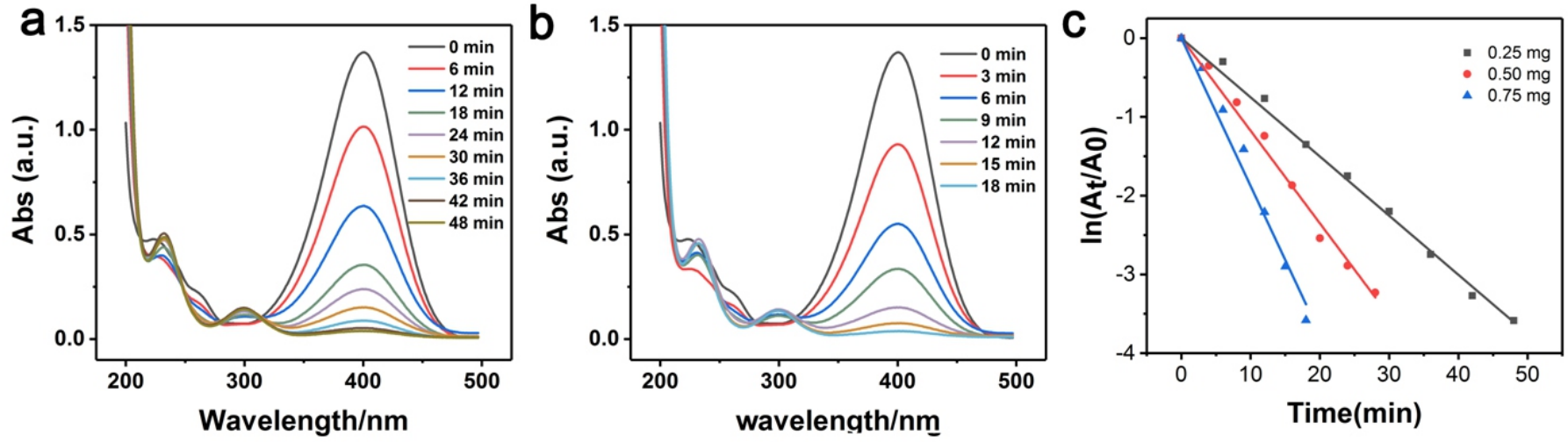

Fig. $8 \mathrm{UV}$ spectrum of (a) $0.25 \mathrm{mg}$, (b) $0.75 \mathrm{mg}$ of Au-NPs/rGO microspheres in $0.4 \mathrm{~mol} 4-\mathrm{NP}$ and $48.0 \mathrm{~mol} \mathrm{NaBH}_{4}$ at $298 \mathrm{~K}$, and the relation of $\mathrm{t}$ vis $\mathrm{ln}$ $\left(\mathrm{At} / \mathrm{A}_{0}\right)(\mathrm{c})$.
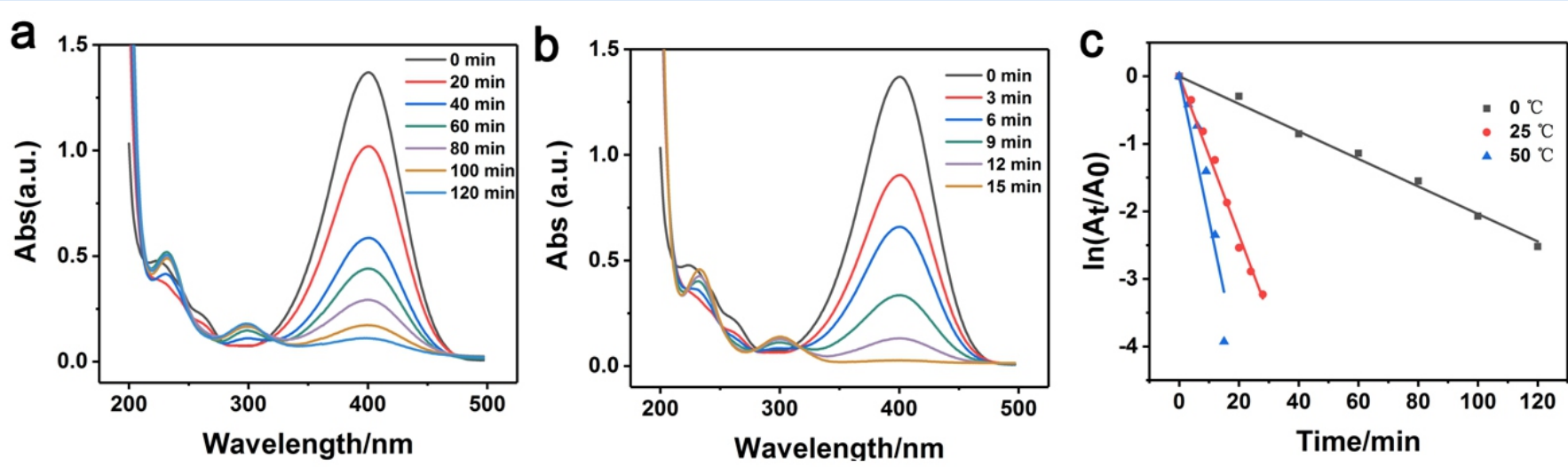

Fig. 9 UV spectrum of Au-NPs/rGO microspheres in $0.4 \mathrm{~mol} 4-\mathrm{NP}$ and $48.0 \mathrm{~mol} \mathrm{NaBH}$ at (a) $273 \mathrm{~K}$, (b) $323 \mathrm{~K}$, and the relation of $\mathrm{t}$ vis $\ln \left(\mathrm{A}_{\mathrm{l}} / \mathrm{A}_{0}\right.$ ) (c) at $273 \mathrm{~K}, 298 \mathrm{~K}$ and $323 \mathrm{~K}$. 
the solution was centrifuged, and the supernatant was used to collect the UV spectrum. The centrifuged substrate was retained, the same amount of 4-NP and $\mathrm{NaBH}_{4}$ were added, and then the same experiment was performed and repeated ten times. Fig. 10 showed the test results, and it was found that the catalytic activities were still retained at $95.1 \%$ after ten cycles. In addition, the reason for the slight loss of performance is possibly due to the incomplete centrifugation or the decreasing catalytic performance of the catalyst itself. These as prepared Au-NPs/rGO microspheres exhibited extraordinary stability as catalysts and demonstrated that our Au-NPs/rGO microsphere can be used as an ideal catalyst toward the reduction of 4-NP reaction and a potential catalytic treatment in environment protection area.

\section{Conclusion}

Novel Au-NPs/reduced graphene oxide microspheres composites were successfully synthesized by the spray-drying technique. The content of $\mathrm{Au}-\mathrm{NPs}$ in the microspheres reached $30.3 \%$. These novel Au-NPs encapsulated within the rGO microsphere exhibited high catalytic activities. The catalytic properties of the Au-NPs within the rGO microspheres towards the reduction of the 4-NP to 4-AP with the presence of the $\mathrm{NaBH}_{4}$ were then investigated, and it was found that first order rate constant was $\mathrm{K}=1.96 \times 10^{-3} \mathrm{~s}^{-1}$, which was measured for the catalytic reduction of $4-\mathrm{NP}$ at given conditions. The activation energy of the reaction, $\mathrm{E}_{\mathrm{a}}=3.5 \times 10^{4} \mathrm{~J} / \mathrm{mol}$, was obtained by using the reaction rate constants at different temperatures. The as-prepared catalysts material can effectively catalyze the reduction of 4-nitrophenol to 4-aminophenol under mild reaction conditions. The stabilities of the catalytic activities of the Au-NPs within rGO microspheres were investigated and it was found that the catalytic activities were maintained $95.1 \%$ after the catalysts were used ten times. This new material exhibited potential applications for the development of novel catalytic device in environment protection and sensor system.

\section{Conflict of interest}

There are no conflicts to declare.

\section{Acknowledgements}

This work was supported by the National Natural Science Foundation

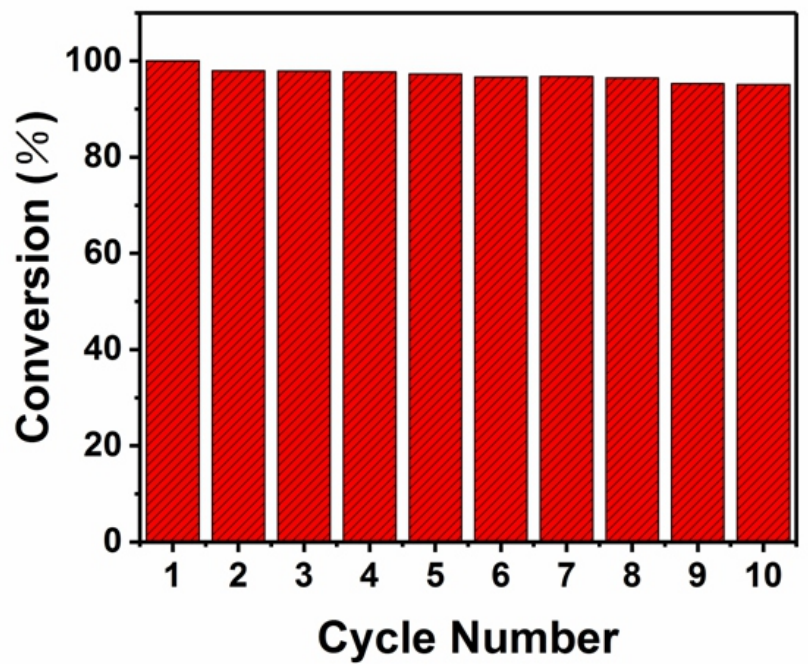

Fig. 10 The catalytic activities towards the reduction of 4-NP to 4-AP in different cycles. of China grant (No. 21475076), International S\&T collaboration Program of China (No. 2015DFA50060), Innovation and achievement transformation projects of Shandong Province (NO. 2014ZZCCX01401).

\section{Reference}

1. K. Kuroda, T. Ishida and M. Haruta, J. Mol. Catal. A-Chem, 2009, 298, 7-11.

2. H. Park, D. A. Reddy, Y. Kim, S. Lee, R. Ma, M. Lim and T. K. Kim, Appl. Surf. Sci, 2017, 401, 314-322.

3. W. Dong, S. Cheng, C. Feng, N. Shang, S. Gao and C. Wang, Catal. Commun, 2017, 90, 70-74.

4. B. Pan, W. Du, W. Zhang, X. Zhang, Q. Zhang, B. Pan, L. Lv, Q. Zhang and J. Chen, Environ. Sci. Technol, 2007, 41, 5057-5062.

5. Ö. S. Kuşcu and D. T. Sponza, Microb. Tech, 2005, 36, 888-895.

6. A. Nezamzadeh-Ejhieh and S. Khorsandi, J. Ind. Eng. Chem, 2014, 20, 937-946.

7. P. Murugaesan, P. Aravind, N. G. Muniyandi and S. Kandasamy, Environ Technol, 2015, 36, 2618.

8. M. Blosi, S. Albonetti, A. L. Costa, N. Sangiorgi and A. Sanson, Chem. Eng. $J, 2013,215(2), 616-625$.

9. R. Krishna, D. M. Fernandes, C. Dias, J. Ventura, E. V. Ramana, C. Freire and E. Titus, Int. J. Hydrogen. Energ, 2015, 40, 4996-5005.

10. K. Wang, X. U. Li, H. Chen, H.Qiao , C. Chen and N. Zhang, Chem. J. Chinese. U, 2016, 37, 723-727.

11. X. Cao, S. Yan, F. Hu, J. H. Wang, Y. Wan, B. Sun and Z. D. Xiao, RSC $A d v, 2016,6,64028-64038$.

12. J. Krajczewski, K. Kołątaj and A. Kudelski, Appl. Surf. Sci, 2016, 388, 624 630.

13. X. Gu, W. Qi, X. Xu, Z. Sun, L. Zhang, W. Liu, X. Pan and D. Su, Nanoscale, 2014, 6(12), 6609-16

14. J. P. Genet, Acc. Chem. Res, 2003, 36(12), 908-918.

15. S. I. Yamazaki, Y. Yamada, S. Takeda, M. Goto, T. Ioroi, Z. Siroma and K. Yasuda, Phys. Chem. Chem. Phys. 2016, 12, 8968-8976.

16. Y. Zheng, J. Shu and Z. Wang, Mater. Lett, 2015, 158, 339-342.

17. X. Jiang, N. Koizumi, X. Guo and C. Song, Appl. Catal. B-Environ, 2015, 170-171, 173-185.

18. K. Mette, S. Kühl, A. Tarasov, H. Düdder, K. Kähler, M. Muhler, R. Schlögl and M. Behrens, Catal. Today, 2015, 242(242), 101-110.

19. J. H. Ramirez, F. J. Maldonado-Hódar, A. F. Pérez-Cadenas, C. MorenoCastilla, C. A. Costa and L. M. Madeira, Appl. Catal. B-Environ, 2007, 75(3-4), 312-323.

20. D. A. Aguilera, A. Perez, R. Molina and S. Moreno, ppl. Catal. B-Environ, 2011, (1-2), 144-150.

21. Y. C. Chang and D. H. Chen, J. Hazard. Mater, 2009, 165(1-3), 664.

22. J. Llorca, A. Casanovas, M. Domínguez, I. Casanova, I. Ngurell and M. Seco, O. Rossell, J. Nanopart. Res, 2007, 10(3), 537-542.

23. M. A. Centeno, M. Paulis, M. Montes and J. A. Odriozola, Appl. Catal. BEnviron, 2005, 61(3-4), 177-183.

24. H. Qi, D. Liu, L. Fei, L. Teng and F. Sun, Rare. Metal. Mat. Eng, 2015, 44, 887-891.

25. A. Bhargava, N. Jain, S. Gangopadhyay and J. Panwar, Process. Biochem 2015, 50, 1293-1300.

26. A. Zhu, Y. Lu, Y. Zhou and S. Dai, Journal of Materials Science: Materials in Medicine, 2010, 21(12), 3095-101.

27. J. Yang, J. Duan ,L. Zhang, B. Lindman, H. Edlund and M. Norgren Cellulose, 2016, 23(5), 3105-15.

28. X. Wang, J. Wang, X. Dong, F. Zhang, L. Ma, X. Fei, X. Zhang and H. Ma, J. Alloy. Compd, 2016, 656, 181-188.

29. Y. Liu, H. Dai, J. Deng, L. Zhang, Z. Zhao, X. Li, Y. Wang, S. Xie, H. Yang and G. Guo, Inorg. Chem. 2013, 52(15), 8665-8676.

30. F. Dong, W. Guo, S. K. Park and C. S. Ha, Chem. Commun, 2011, 48, 11081110.

31. A. K. Geim and K. S. Novoselov, Nat. Mater, 2007, 6, 183-191.

32. S. Stankovich, D. A. Dikin, R. D. Piner, K. A. Kohlhaas, A. Kleinhammes and Y. Jia, Carbon. 2007, 45(7), 1558-65.

33. B. Vellaichamy, P. Prakash and J. Thomas, Ultrasonics Sonochemistry, 2018 , 48, 362-9. 
34. Z. Liu, J. T. Robinson, X. Sun and H. Dai, J. Am. Chem. Soc, 2008, 130, 10876-10877.

35. G. Prieto, H. Tüysüz, N. Duyckaerts, J. Knossalla , G. H. Wang and F. Schüth, Chem. Rev., 2016, 116(22), 14056-119.

36. F. Zhang, Y. Yuan, Y. Zheng, H. Wang, T. Liu and S. Hou, Microchim. Acta, 2017,184, 1565-1572.

37. M. Šimšíková, M. Bartoš, P. Keša and T. Šikola, Mater. Chem. Phys., 2016, 177, 339-45.

38. B. Wang, S. Yan and Y. Shi, J. Solid. State. Electr, 2015, 19, 307-314.

39. J. M. Walker and J. M. Zaleski, Nanoscale, 2015, 8(3), 1535-1544.

40. P. Zhao, X. Feng, D. Huang and G. Yang, Coordin. Chem. Rev, 2015, 287, $114-136$

41. K. B. Narayanan and N. Sakthivel, J. Hazard. Mater, 2011, 189, 519-25.

42. H. Fang, M. Wen, H. Chen, Q. Wu and W. Li, Nanoscale, 2015, 8, 536-542.
43. Z. Zhang, C. Shao, Y. Sun, J. Mu, M. Zhang, P. Zhang, Z. Guo, P. Liang, C. Wang and Y. Liu, J. Mater. Chem, 2011, 22, 1387-1395.

44. L. F. Villalobos, P. Neelakanda, M. Karunakaran, D. Cha and K. V. Peinemann, Catal. Today, 2014, 236, 92-97.

45. J. Luo, N. Zhang, R. Liu and X. Liu, RSC. Adv, 2014, 4, 64816-64824.

46. Q. Wang, W. Jia, B. Liu, A. Dong, X. Gong, C. Li, P. Jing, Y. Li, G. Xu and J. Zhang, J. Mater. Chem. A, 2013, 1, 12732-12741.

47. Y. Choi, H. S. Bae, E. Seo, S. Jang, K. H. Park and B. S. Kim, J. Mater. Chem, 2011, 21, 15431-15436

48. T. Ji, L. Li, M. Wang, Z. Yang and X. Lu, RSC Adv., 2014, 4(56), 29591-4.

49. S. Panigrahi, S. Basu, S. Praharaj, S. Pande, S. Jana, A. Pal, S. Kuma. R. Ghosh and P. Tarasankar, J. Phys. Chem. C, 2007, 111, 4596-4605.

Publisher's Note Engineered Science Publisher remains neutral with regard to jurisdictional claims in published maps and institutional affiliations. 